\title{
COMPARACIÓN DE LA EFECTIVIDAD DE DOS ESTRATEGIAS FARMACOLÓGICAS PARA LA PREVENCIÓN DEL SÍNDROME DE IRIS FLÁCIDO INTRAQUIRÚRGICO
}

\section{COMPARISON OF THE EFFECTIVENESS OF TWO DIFFERENT PHARMACOLOGIC APPROACHES TO PREVENT INTRAOPERATIVE FLOPPY IRIS SYNDROME}

\author{
PÉREZ-SILGUERO D ${ }^{1}$, RAMALLO-FARIÑA Y ${ }^{2}$, PÉREZ-SILGUERO MA ${ }^{1}$, JIMÉNEZ-GARCÍA A ${ }^{3}$, \\ PEÑATE-VALDIVIELSO ${ }^{3}$, PÉREZ-HERNÁNDEZ FR ${ }^{1}$
}

\section{RESUMEN}

Objetivo: Comparar la efectividad de dos estrategias diseñadas para prevenir la aparición del síndrome de iris flácido intraquirúrgico (IFIS) en pacientes expuestos a $\alpha$-bloqueantes.

Métodos: Comparamos retrospectivamente la presencia y severidad de aparición del síndrome en 29 pacientes intervenidos de cataratas, estableciendo dos grupos de 12 pacientes ( 22 ojos) y 17 pacientes (33 ojos). La estrategia aplicada al grupo uno consistió en suspender le exposición a los $\alpha$-bloqueantes un mínimo de una semana antes de la intervención, atropina tópica $1 \% 3$ veces al día desde dos días antes, y adrenalina intracamerular. La estrategia del grupo dos fue la suspensión del fármaco un mínimo de una semana antes y la aplicación intracamerular al inicio de la cirugía de una solución formada por suero fisiológico, adrenalina y lidocaína.

Resultados: En el grupo uno 6 pacientes no tuvieron IFIS, 2 padecieron IFIS leve bilateral, 2 moderado bilateral y 2 severo (unilaterales); es decir, un $45,5 \%$ de ojos lo padecieron. En el grupo dos, 3

\section{ABSTRACT}

Objective: To compare the effectiveness of two strategies to prevent the appearance of intraoperative floppy iris syndrome (IFIS) in patients exposed to $\alpha$-blockers.

Methods: We compared retrospectively the presence and severity of the appearance of IFIS in 29 patients subjected to cataract surgery, establishing two groups of 12 patients ( 22 eyes) and 17 patients (33 eyes). The strategy applied to group one consisted of suspending the exposure to $\alpha$-blockers for at least one week prior to the intervention, the topical application of $1 \%$ atropine three times a day for two days before the intervention and the use of intracameral lidocaine. The strategy for group two consisted of suspending use of the drug for at least one week before the intervention and the intracameral application at the beginning of surgery of a solution of physiological serum, adrenaline and lidocaine.

Results: Six patients from group one showed no evidence of IFIS, 2 exhibited bilateral mild symptoms, 2 bilateral moderate and 2 unilateral

\footnotetext{
Recibido: 23/6/08. Aceptado: 25/11/09.

Sección de polo anterior. Centro Insular de Oftalmología (CIOF). Las Palmas de Gran Canaria. España.

1 Doctor en Medicina.

${ }^{2}$ Licenciado en Medicina. Servicio de Bioestadística del Servicio Canario de Salud.

${ }^{3}$ Licenciado en Medicina.

Correspondencia:

David Pérez Silguero

C/. Obispo Rabadán, 18

35003 Las Palmas de Gran Canaria

España

E-mail: dpsilguero@msn.com
} 
ojos $(9,1 \%)$ de dos pacientes lo padecieron en grado leve. Existen diferencias estadísticamente significativas en los dos grupos ( $\mathrm{p}=0,002)$. Si consideramos que un paciente sufre el síndrome sólo cuando tiene un grado moderado o grave, también existen diferencias significativas entre la proporción de casos con IFIS en el grupo uno con respecto al grupo dos $(\mathrm{p}=0,0002)$.

Conclusiones: El estudio demuestra la mayor efectividad estadísticamente significativa de la estrategia aplicada en el grupo dos comparada a la aplicada al grupo uno.

Palabras clave: Iris flácido, $\alpha$-bloqueantes, miosis progresiva, prolapso de iris, hipertrofia de próstata. severe symptoms of IFIS. In total, $45.5 \%$ eyes suffered from IFIS. In group two, only 3 eyes $(9.1 \%)$ from 2 patients exhibited mild symptoms. Statistical analysis showed a significant difference between both groups $(\mathrm{p}=0.002)$. If only those exhibiting moderate and severe symptoms are taken into account the result was highly statistically significant $(\mathrm{p}=0.0002)$.

Conclusion: The study shows a greater statistical effectiveness of the strategy applied to group two compared to the strategy applied to group one (Arch Soc Esp Oftalmol 2009; 84: 549-556).

Key words: Floppy iris, $\alpha$-blockers, progressive miosis, prolapse of the iris, prostatic hypertrophy.

\section{INTRODUCCIÓN}

El Síndrome de Iris Flácido Intraoperatorio (IFIS) fue descrito por Chang y Campbell (1) en 2005. Acontece durante la cirugía de cataratas, y se caracteriza por la aparición de la triada: estroma iridiano flácido que ondula en respuesta a la irrigación intraocular, una propensión del mismo a prolapsarse hacia la punta del facoemulsificador y las incisiones, y una constricción pupilar progresiva a pesar de la utilización de medidas farmacológicas preoperatorias estándar (1). Se asocia al uso de bloqueantes $\alpha-1$ adrenérgicos sistémicos, sobre todo tamsulosina, utilizados en la hipertrofia benigna de próstata y la hipertensión arterial. Son varias las metodologías propuestas para evitar su aparición o facilitar su manejo: prequirúrgicas, farmacológicas intraoperatorias, el uso de dispositivos mecánicos intraoperatorios, y la variación de los parámetros que controlan la fluídica intraquirúrgica.

Nos hemos propuesto comparar dos estrategias farmacológicas preventivas.

\section{SUJETOS, MATERIAL Y MÉTODOS}

Estudio retrospectivo de 29 pacientes (55 ojos) afectos de catarata, distribuidos en dos grupos, intervenidos desde octubre de 2006 hasta mayo de 2008, en tratamiento con $\alpha-1$ bloqueantes, principalmente tamsulosina, pero también otros como doxazosina ( 8 ojos), terazosina (2 ojos) y alfuzosina (4 ojos), con el fin de valorar dos estrategias farmacológicas preventivas del IFIS.
La primera consiste en suspender $\alpha-1$ bloqueantes desde un mínimo de una semana antes de la cirugía, administrar gotas de atropina $1 \% 3$ veces al día desde dos días antes, dilatar el día de cirugía con tropicamida, ciclopléjico y fenilefrina, y utilizar adrenalina intracamerular 1/5000 tras la realización de la paracentesis de servicio. En la segunda estrategia se procedió de igual forma con la medicación oral, no se aplicó atropina tópica, la dilatación pupilar el día de la cirugía fue idéntica a la descrita, y tras la paracentesis se inyectó $0,2-0,3 \mathrm{ml}$ de una mezcla farmacológica formada por $4,5 \mathrm{ml}$ de BSS, $2 \mathrm{ml}$ de adrenalina (1/3000), y 1,5 ml de lidocaína al 5\%, dejándola actuar 30 segundos antes de continuar. Durante el proceso los pacientes estaban monitorizados y vigilados por un anestesista.

Las cirugías fueron realizadas por el mismo y experimentado cirujano (DPS), sin variar su técnica quirúrgica habitual («Phaco-cracking: the ultimate surgery in cataratact», presentado en el Continuous Video Programme de la European Society of Cataract and Refractive Surgeons, Bruselas, septiembre 2000) aplicando especial cuidado en la arquitectura correcta de la incisión, siempre en córnea clara de 2,75 mm, intentando además enlentecer la fluídica en el momento de la hidrodisección. En los pacientes bilaterales la cirugía de ambos ojos se realizó en la misma sesión quirúrgica, como hacemos de manera habitual en nuestro centro.

Desde enero de 2006 hemos protocolizado dos tablas que son completadas de manera rutinaria en todos los pacientes que estén expuestos a $\alpha 1$-bloqueantes y sean intervenidos de cataratas; reflejan el grado de dilatación pupilar previa (tabla I) y el 
Tabla I. Grado de dilatación pupilar previa a cirugía aplicado en nuestro estudio

\begin{tabular}{lc}
\hline Dilatación previa & Diámetro pupilar en mm \\
\hline Buena & 9 o más \\
Media & $7-8$ \\
Mala & 6 o menos \\
\hline \hline
\end{tabular}

grado de IFIS sufrido en la cirugía (tabla II). En todas las cirugías se utilizó Infiniti ${ }^{\mathrm{TM}}$ visión system con tecnología Ozyl torsional (laboratorios Alcon, Irvine, CA, EEUU): Altura de la botella de $110 \mathrm{~cm}$ $\mathrm{H}_{2} 0$, vacío de $320 \mathrm{mmHg}$, aspiración de $32 \mathrm{cc} / \mathrm{min}$. Si existía algún signo que indicase presencia de IFIS moderado variábamos a $90 \mathrm{~cm}$ de altura, vacío de 230 y aspiración de 23 .
El grupo uno lo forman 12 pacientes (22 ojos), 10 bilaterales y 2 unilaterales (tabla III), de los cuales uno de ellos estaba programado para cirugía bilateral pero se decidió postponer la cirugía del ojo adelfo tras sufrir IFIS severo en el primer ojo intervenido. Todos los pacientes del grupo dos (17 pacientes, 33 ojos) presentaban catarata bilateral (tabla IV), aunque al paciente 17 sólo se le aplicó la prevención en el OI, por lo que no contabilizamos para el estudio el OD en el que sufrió IFIS severo (no conocíamos la exposición del paciente a los $\alpha$-bloqueantes).

Se excluyeron aquellos pacientes con patologías que pudieran afectar al grado de dilatación pupilar antes o durante la cirugía (patologías neurológicas, retinopatía diabética en grado moderado o superior, glaucoma, cirugías intraoculares, historia de traumatismos perforantes, uveítis, heterocromías no filiadas).

Tabla II. Gradación de IFIS aplicada en nuestro estudio

\begin{tabular}{|c|c|c|c|c|c|c|}
\hline \multicolumn{7}{|c|}{ Hallazgos Quirúrgicos } \\
\hline & $\begin{array}{c}\text { Miosis } \\
\text { progresiva }\end{array}$ & Prolapso de Iris & $\begin{array}{l}\text { Cambios } \\
\text { en Fluidica }\end{array}$ & $\begin{array}{l}\text { Uso específico } \\
\text { de viscoelástico }\end{array}$ & $\begin{array}{l}\text { Complicaciones del } \\
\text { síndrome derivadas }\end{array}$ & Hallazgos postquirúrgicos \\
\hline No IFIS & No & No & No necesarios & No necesario & No & No \\
\hline IFIS leve & Moderada & $\begin{array}{l}\text { Esporádico,casi } \\
\text { siempre en fase } \\
\text { de irrigación/ } \\
\text { aspiración }\end{array}$ & No necesarios & No necesario & No & No \\
\hline IFIS moderado & Moderada & $\begin{array}{l}\text { Desde la fase } \\
\text { de hidrodisección }\end{array}$ & $\begin{array}{l}\text { Necesarios, } \\
\text { y son eficaces }\end{array}$ & $\begin{array}{l}\text { Necesario, } \\
\text { y es eficaz }\end{array}$ & $\begin{array}{l}\text { Potencialmente, } \\
\text { manejables en } \\
\text { manos expertas }\end{array}$ & $\begin{array}{l}\text { Áreas hipopigmentadas } \\
\text { y traslúcidas en el estroma } \\
\text { iridiano }\end{array}$ \\
\hline IFIS severo & Severa & $\begin{array}{l}\text { Desde la fase } \\
\text { de hidrodisección }\end{array}$ & $\begin{array}{l}\text { Necesarios, } \\
\text { poco eficaces }\end{array}$ & $\begin{array}{l}\text { Necesario, } \\
\text { poco eficaz }\end{array}$ & Sí & $\begin{array}{l}\text { Pérdida de sustancia } \\
\text { del estroma iridiano y } \\
\text { los derivados de las } \\
\text { complicaciones } \\
\text { potenciales añadidas }\end{array}$ \\
\hline
\end{tabular}

Tabla III. Grupo 1, formado por los pacientes a quienes se aplicó la estrategia 1

\begin{tabular}{|c|c|c|c|c|c|c|}
\hline Pacientes & Bloqueante alfa-1 & $\begin{array}{l}\text { Suspensión tto } \\
\text { antes cirugía (días) }\end{array}$ & Patología sistémica & $\begin{array}{l}\text { Dilatación } \\
\text { previa }\end{array}$ & Resultado & Cirugía \\
\hline 1 & Tamsulosina & 15 & Cardiópata, DM & Media & IFIS leve & bilateral \\
\hline 2 & Tamsulosina & 15 & Cardiópata, DM & Mala & No IFIS & bilateral \\
\hline 3 & Tamsulosina & 7 & Cardiópata, DM, HTA & Mala & IFIS moderado & bilateral \\
\hline 4 & Tamsulosina & 15 & Cardiópata, DM, HTA & Media & IFIS leve & bilateral \\
\hline 5 & Doxazosina & 7 & HTA, asma & Buena & No IFIS & bilateral \\
\hline 6 & Tamsulosina & 15 & HTA. & Buena & No IFIS & bilateral \\
\hline 7 & Tamsulosina & 10 & No patología & Media & No IFIS & bilateral \\
\hline 8 & Doxazosina & 7 & No patología & Buena & No IFIS & bilateral \\
\hline 9 & Tamsulosina & 15 & Cardiópata, HTA & Buena & IFIS moderado & bilateral \\
\hline 10 & Tamsulosina & 7 & HTA & Media & IFIS severo & OD \\
\hline 11 & Tamsulosina & 15 & $\mathrm{DM}$ & Buena & IFIS severo & OD \\
\hline 12 & Tamsulosina & 15 & DM & Buena & No IFIS & bilateral \\
\hline
\end{tabular}

HTA: Hipertensión arterial; DM: Diabetes mellitus. 
Tabla IV. Grupo 2, pacientes a quienes se les aplicó la estrategia 2

\begin{tabular}{|c|c|c|c|c|c|c|}
\hline Pacientes & Bloqueante alfa-1 & $\begin{array}{l}\text { Suspensión tto } \\
\text { antes cirugía (días) }\end{array}$ & Patología sistémica & $\begin{array}{c}\text { Dilatación } \\
\text { previa }\end{array}$ & Resultado & Cirugía \\
\hline 1 & Tamsulosina & 365 & Cardiópata,HTA & Buena & No IFIS & Bilateral \\
\hline 2 & Alfuzosina & 7 & DM & Buena & No IFIS & Bilateral \\
\hline 3 & Tamsulosina & 7 & Cardiópata, HTA, DM & Buena & No IFIS & Bilateral \\
\hline 4 & Tamsulosina & 15 & HTA & Buena & No IFIS & Bilateral \\
\hline 5 & Tamsulosina & 7 & DM, broncópata & Buena & No IFIS & Bilateral \\
\hline 6 & Terazosina & 15 & Cardiópata, HTA & Mala & No IFIS & Bilateral \\
\hline 7 & Doxazosina & 7 & HTA & Mala & No IFIS & Bilateral \\
\hline 8 & Tamsulosina & 10 & HTA & Buena & No IFIS & Bilateral \\
\hline 9 & Tamsulosina & 10 & DM & Media & No IFIS & Bilateral \\
\hline 10 & Tamsulosina & 7 & $\mathrm{DM}$ & Buena & No IFIS & Bilateral \\
\hline 11 & Tamsulosina & 7 & Cardiópata, HTA & Buena & No IFIS & Bilateral \\
\hline 12 & Tamsulosina & 7 & Broncópata & Media & No IFIS & Bilateral \\
\hline 13 & Doxazosina & 7 & HTA & Buena & No IFIS & Bilateral \\
\hline 14 & Alfuzosina & 15 & No patología & Buena & No IFIS & Bilateral \\
\hline 15 & Tamsulosina & 7 & $\mathrm{DM}$ & Media & No IFIS & Bilateral \\
\hline 16 & Tamsulosina & 7 & Broncópata & Buena & IFIS leve & Bilateral \\
\hline 17 & Tamsulosina & 15 & $\mathrm{DM}$ & Media & IFIS leve & $\begin{array}{l}\text { Unilateral, } \\
\text { tto sólo en OI }\end{array}$ \\
\hline
\end{tabular}

Tto=tratamiento preventivo aplicado; HTA: Hipertensión Arterial; DM: Diabetes Mellitus.

Para el análisis estadístico, se han utilizado la Chi- cuadrado de Pearson y la prueba exacta de Fisher, con un nivel de significación de 0,05 utilizando el software estadístico SPSS 12.0, tomando como unidad de análisis el ojo. Las potencias de los test se han calculado con el StudySize 2.0.4.

\section{RESULTADOS}

Desde octubre de 2006 hasta abril de 2008 se intervinieron 566 pacientes varones (1.111 pacientes teniendo en cuenta ambos sexos), de los cuales 35 estaban bajo tratamiento con $\alpha$-bloqueantes, lo que supone un 6,18\% de los pacientes varones intervenidos de cataratas. Cuatro de ellos fueron desestimados para el estudio por presencia de glaucoma mal controlado y 2 por retinopatía diabética. A 12 pacientes (22 ojos) se les aplicó la estrategia uno (tabla III) y a 17 (33 ojos) la estrategia dos (tabla IV).

En el grupo uno 6 pacientes no tuvieron IFIS, 2 padecieron IFIS leve bilateral, 2 moderados bilateral y 2 severos (pacientes unilaterales); es decir, un $45,5 \%$ de ojos lo padecieron (50\% de pacientes), y teniendo en cuenta los casos moderados y severos (es decir, aquellos que afectarían de manera significativa al correcto transcurso de la cirugía) se trataría de un 27,3\% (33\% de pacientes). En el grupo dos, un paciente sufrió el síndrome bilateral, y otro unilateral, ambos en grado leve. Este último presentó IFIS severo en OD sin tratamiento preventivo por desconocimiento de los antecedentes de exposición a tamsulosina y en el OI se aplicó la prevención sufriendo IFIS leve (figs. 1 y 2). La comparación de la distribución de los diferentes grados del síndrome (tabla V) establece diferencias estadísticamente significativas en los dos grupos ( $\mathrm{p}=0,002)$. Si consideramos que un paciente sufre el síndrome sólo

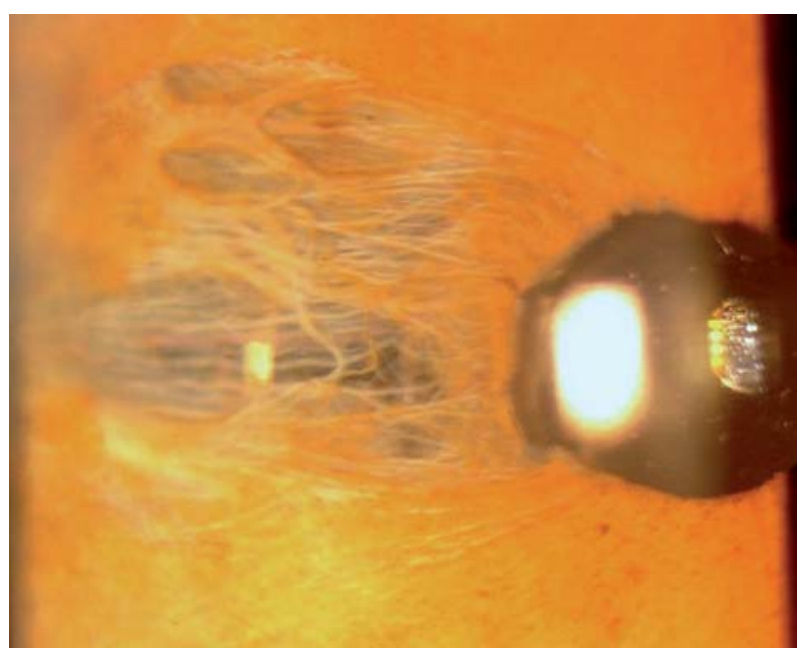

Fig. 1: Atrofia iridiana tras cirugía en IFIS severo en paciente sin estrategia de prevención. 


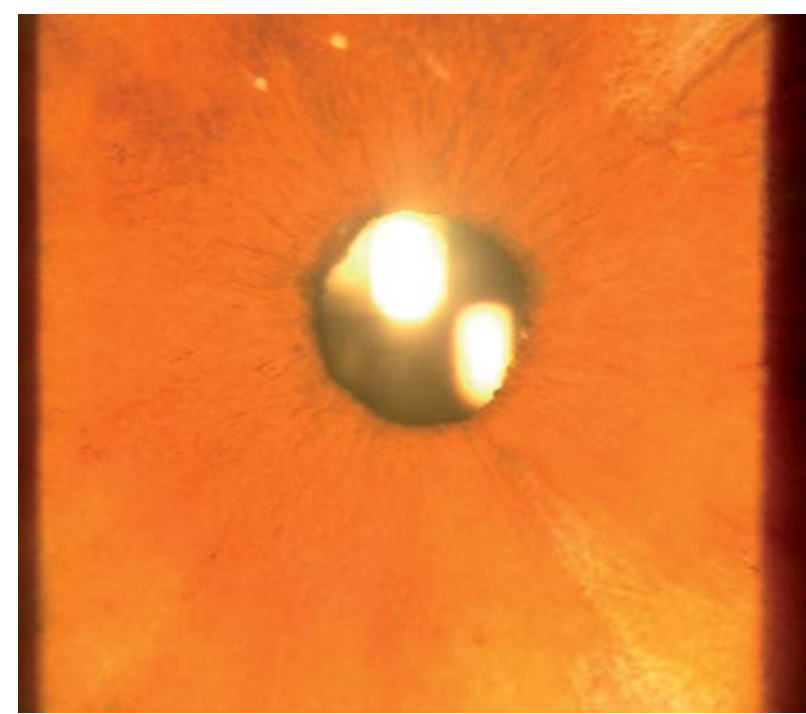

Fig. 2: Ojo adelfo del paciente de la fig. 1 tras aplicar la estrategia del grupo 2.

Tabla V. Número de ojos según estrategia de prevención, clasificación de IFIS y Prueba exacta de Fisher*

\begin{tabular}{|c|c|c|c|c|}
\hline \multirow[b]{2}{*}{ No IFIS } & $\begin{array}{c}\text { Grupo } 1 \\
\mathrm{~N}(\%)\end{array}$ & \multicolumn{2}{|c|}{$\begin{array}{c}\text { Grupo } 2 \\
\mathrm{~N}(\%)\end{array}$} & \multirow{2}{*}{$\begin{array}{c}\begin{array}{c}\text { Significación } \\
\text { estadística }\end{array} \\
\left.\mathrm{p}^{*}\right)=0,002\end{array}$} \\
\hline & $(54,5)$ & 30 & $(90,9)$ & \\
\hline IFIS leve & $4 \quad(18$, & 3 & $(9,1)$ & \\
\hline IFIS moderado & $4 \quad(18,2)$ & 0 & $(0,0)$ & \\
\hline IFIS s & $2 \quad(9,1)$ & 0 & $(0,0)$ & \\
\hline Total ojos & $22(100)$ & 33 & (100) & \\
\hline
\end{tabular}

cuando tiene un grado moderado o grave, existen diferencias significativas entre la proporción de casos con IFIS en el grupo 1 con respecto al grupo $2(\mathrm{p}=0,003)$.

En todos los pacientes intervenidos bilateralmente que presentaron IFIS, el síndrome aconteció en ambos ojos y con la misma intensidad.

\section{DISCUSIÓN}

La mitad de los varones mayores de 50 años y el $90 \%$ de los que sobrepasan los 80 años de edad pre- sentan hipertrofia benigna de próstata (4). Si unimos a estas cifras las de los pacientes hipertensos que ingieren $\alpha$-bloqueantes, nos podemos dar cuenta de la magnitud del problema; son muchas las complicaciones que pueden acontecer cuando nos enfrentamos a este síndrome en su grado más severo, como atrofia sectorial iridiana severa y diplopía monocular, edema macular quístico, dehiscencia zonular, hipertensión ocular postquirúrgica, rotura de cápsula posterior con o sin vitreorragia, hiphema, entre otras $(5,6)$; En nuestra serie los casos severos se solucionaron con la única complicación postquirúrgica de la existencia de atrofia sectorial de iris en todos ellos (fig. 1) pero sin diplopía. Según algunos trabajos, un 4\%-7\% de los pacientes sometidos a cirugía de cataratas toman $\alpha$-bloqueantes $(5,7,8)$. En nuestro caso un $6,18 \%$ de los varones lo tomaban, lo que supone un porcentaje de 3,15\% del total de pacientes (hombres y mujeres) intervenidos en ese período (1.111 pacientes). La diferencia en la prevalencia del uso de $\alpha$-bloqueantes se explica por las variaciones geográficas en la incidencia de hipertrofia prostática y en el algoritmo de tratamiento de la misma (5). De nuestros pacientes 7 ingerían $\alpha$-bloqueantes diferentes a la tamsulosina. Chang y Campbell (1) estimaron la prevalencia de síndrome IFIS en la población sometida a cirugía de cataratas en un $2 \%$. Otros estudios se mueven en un rango entre $0,6-3 \%(1,7,9,10)$. Los varones expuestos a la tamsulosina desarrollan el síndrome en un porcentaje que va desde $57 \%$ al $100 \%$ $(1,5,7,9,10)$. Debido a que se trata de estudios sin estrategia de prevención, no son comparables con nuestro trabajo, en el que lo que se pretende es comparar la eficacia entre diferentes aproximaciones farmacológicas en aras a prevenir la aparición del mismo; sin embargo son cifras que se asemejan a lo ocurrido en el grupo uno si tenemos en cuenta todos los grados de IFIS: $45,45 \%$ de ojos (50\% de pacientes); si nos atenemos a los casos moderados y severos se trataría de un $27,27 \%$ ( $33 \%$ de pacientes).

El síndrome tiene una clara descripción con 3 parámetros expuestos por Chang (1). Sin embargo, dicha descripción se basa en casos analizados sin la

Tabla VI. Muestra el número de casos de IFIS dependiendo del grado de dilatación previa

\begin{tabular}{lccccc}
\hline & & No IFIS & IFIS Leve & IFIS Moderado & IFIS severo \\
\hline Dilatación Buena & 3 pacientes & 0 & 0 & 2 pacientes (bilateral) & 1 paciente (unilateral) \\
Dilatación Media & 4 pacientes & 1 & 2 & 1 & 1 paciente (unilateral) \\
Dilatación Mala & 2 pacientes & 1 & & 1 & \\
\hline \hline
\end{tabular}


existencia de ninguna estrategia de prevención. Una vez introducidos parámetros nuevos, como la atropina tópica, o la adrenalina intracamerular, los signos descritos originalmente disminuyen en magnitud o desaparecen; creemos que la gradación que hemos aplicado (tabla II) refleja lo acontecido durante estas cirugías. Otros autores también establecieron su gradación: Cheung et al (11) y Chadha et al (7), quienes hablan de IFIS completo o incompleto con una puntuación de 0 a 3 , según el número de signos de la triada que se observe. Takmaz et al dan por hecho que la severidad del IFIS es variable, presentándose en varios grados incluso en los ojos de un mismo paciente (6).

De los $\alpha$-bloqueantes, el que más predispone a la aparición de IFIS es la tamsulosina: De un $80 \%$ a un $86,4 \%$ de los casos reportados se deben a ella $(5,8)$. Los únicos $\alpha$-bloqueantes $\alpha 1$ selectivos son la tamsulosina y la alfuzosina, siendo la tamsulosina el único $\alpha 1 \mathrm{~A}$ selectivo, receptor que predomina en el iris (5). No parece existir relación con la dosis del fármaco (8), ni con el tiempo de exposición al mismo (5), existiendo casos con sólo 3 meses de exposición (11). Además los efectos sobre el iris podrían ser incluso irreversibles: existen casos en que la ingesta del fármaco se suspendió 1 año antes (con exposición de sólo 4 meses) (11) e incluso 3 años antes (1), con idéntico resultado: Presencia de IFIS.

En nuestro trabajo todos los pacientes que presentaron IFIS lo hicieron de manera similar en ambos ojos, incluso en cuanto a la severidad del mismo. Sin embargo, no es infrecuente encontrar en la literatura casos asimétricos, o que acontezca en un ojo y no en el otro $(5,6,8)$. Esta diferencia con respecto a nuestros casos pudiera deberse a que nuestras cirugías se realizaron de manera bilateral consecutiva, a diferencia de las otras casuísticas publicadas; ello podría sugerir que existen causas multifactoriales que influyen en su presencia, que pueden variar dependiendo del momento de la cirugía, coincidiendo con lo expresado por Srinivasan et al (8).

Con respecto a la relación existente entre el IFIS y las enfermedades que se asocian con disregulación endotelial (diabetes mellitus e hipertensión arterial), algunos trabajos no encuentran asociación estadística al estudiar dicha relación $(5,7)$ aunque otros sí parecen demostrarla $(12,8)$. En nuestro trabajo el tamaño muestral es insuficiente para que la potencia del test sea significativa. Aún así, y coincidiendo con otros autores (5) que tampoco han podi- do demostrarlo estadísticamente, nuestra experiencia parece indicar la existencia de una tendencia a aumentar el IFIS en estos pacientes; sin embargo sí encontramos asociación estadística significativa entre los pacientes del grupo 1 con cardiopatía y la aparición del síndrome, tomando como unidad de análisis el ojo ( $\mathrm{p}=0,008$; potencia del $98 \%$ ), aunque es un dato que debe sopesarse con prudencia y valorarlo con series más amplias.

No hemos encontrado estudios que relacionen la dilatación previa y la presencia y grado del síndrome, aunque algunos autores exponen que los casos más graves de IFIS parecen suceder en pacientes con mala dilatación previa (14). En nuestros casos parece no existir relación entre ambas variables, incluso estadísticamente no existen suficientes evidencias con un nivel de significación de 0,05 para rechazar que la aparición del síndrome y la dilatación previa son independientes $(\mathrm{p}=0,200$; potencia $=6 \%$ ), aunque el tamaño de la muestra es insuficiente para que la potencia del test sea significativa (tabla VI). Aclarar que los 2 pacientes con mala dilatación del grupo dos no sufrieron IFIS, pero no estaban expuestos a tamsulosina, sino a doxazosina y terazosina, y que todos los casos de mala dilatación se encontraban por encima de los $5 \mathrm{~mm}$ de diámetro pupilar.

Farmacológicamente son varias las estrategias descritas para enfrentarse a este síndrome, comenzando por aconsejar la eliminación de la exposición a $\alpha$-bloqueantes (6), aunque ya hemos visto que no se sabe si realmente esta medida contribuye a algo. La introducción de la atropina tópica desde 2-3 días antes de la cirugía o incluso horas antes parece ayudar a dar más tensión al iris $(2,13)$, aunque otros autores discrepan e incluso lo desaconsejan (14). El uso de epinefrina intracamerular se basa en la afinidad de la epinefrina por los $\alpha 1$ receptores, ayudando a dilatar y contribuyendo a dar tono al iris. Se aplica al inicio de la cirugía, repitiéndola en diferentes fases de la misma, o introduciéndola en el suero de infusión, a diferentes dosis según los autores, desde $1 / 10000$ hasta $1 / 2500(2,15-17)$.

Nosotros decidimos inicialmente adoptar la estrategia aplicada al grupo uno: consistía en eliminar la exposición al fármaco, aplicar atropina tópica $1 \% 2$ días antes de la cirugía, 3 veces al día, y utilizar adrenalina 1/5000 intracamerular. Sin embargo no obtuvimos resultados esperanzadores (tabla III), y decidimos adoptar otra postura: eliminamos la atropina porque podría contribuir a la 
retención urinaria de pacientes a los que suspenderíamos el $\alpha$-bloqueante; y al comienzo de la cirugía introdujimos vía intracamerular 0,1-0,2 $\mathrm{ml}$ de una solución con suero fisiológico (4,5 cc), lidocaína $(1,5 \mathrm{cc}$ al $5 \%)$ y adrenalina (1/3000), con monitorización electrocardiográfica de los pacientes. La lidocaína nos ayudaba a conseguir el confort analgésico del paciente y a mantener la dilatación $(3,18,19)$. Decidimos entonces comparar ambas estrategias, que es el objetivo del presente trabajo, con la evidente superioridad de la estrategia utilizada en el grupo dos, posiblemente debida a una supuesta sinergia entre la acción dilatadora y aumento de tono iridiano que producen la lidocaína y adrenalina. En la revisión bibliográfica hemos encontrado 2 trabajos con estrategia similar a la que aplicamos al grupo dos: en una de ellas (2) se utiliza la atropina tópica desde días antes, y se especifica que el éxito de la estrategia se basa en la sinergia de los efectos entre atropina y epinefrina (1/2500), inyectando lidocaína $1 \%$ antes de los viscoelásticos, y la epinefrina después de los mismos, y bajo el iris; en la otra (14) utilizan epinefrina al 1/1000, lidocaína al $4 \%$ y BSS-plus (Alcon, Irvine, CA, EEUU) (14), dando importancia crucial al hecho de que la solución balanceada sea plus, ya que el pH del mismo contribuye a su eficacia en este síndrome: además no observan ventaja en el uso de atropina. Nosotros utilizamos suero fisiológico en vez de BSS y los resultados son similares: en el grupo dos un paciente sufrió IFIS leve bilateral y otro paciente IFIS leve unilateral (en el adelfo no se aplicó la prevención). En ambos casos la medicación preventiva no impidió la miosis progresiva, pero sí el prolapso de iris. En ninguno de nuestros pacientes la adrenalina provocó elevaciones de la tensión arterial ni variaciones en la frecuencia que necesitasen tratamiento específico.

Concluimos expresando la mayor efectividad obtenida con la estrategia utilizada en el grupo dos con respecto a la utilizada en el grupo uno. Actualmente es la estrategia aplicada por nuestro equipo en todos los casos en los que nos enfrentamos a pacientes expuestos a cualquier $\alpha$-bloqueante.

\section{BIBLIOGRAFÍA}

1. Chang DF, Campbell JR. Intraoperative floppy iris syndrome associated with tamsulosin. J Cataract Refract Surg 2005; 31: 664-673.
2. Masket S, Belani S. Combined preoperative topical atropine sulfate $1 \%$ and intracameral nonpreserved epinephrine hydrochloride 1:2500 for management of intraoperative floppy-iris syndrome. J Cataract Refract Surg 2007; 33: 580-582.

3. Nikeghbali A, Falavarjani KG, Kheirkhah A, Bakhtiari P, Kashkouli MB. Pupil dilation with intracameral lidocaina during phacoemulsification. J Cataract Refract Surg 2007; 33: 101-103.

4. Ziada A, Rosemblum M, Crawford ED. Benign prostatic hyperplasia: an overview. Urology 1999; 53: 1-6.

5. Blouin MC, Blouin J, Perreault S, Lapointe A, Dragomir A. Intraoperative floppy-iris syndrome associated with $\alpha 1$ adrenorreceptores. J Cataract Refract Surg 2007; 33: 1227-1234.

6. Takmaz T, Can I. Intraoperative floppy-iris syndrome: Do we know everything about it? J Cataract Refract Surg 2007; 33:1110-1112.

7. Chadha V, Borooah S, Tey A, Styles C, Singh J. Floppy iris bahaviour during cataract surgery: associations and variations. Br J Ophthalmol 2007; 91: 40-42.

8. Srinivasan S, Radomski S, Chung J, Plazker T, Singer S, Slomovic AR. Intraoperative floppy iris syndrome during cataract surgery in men using alpha-blockers for benign prostatic hypertrophy (correspondence). J Cataract Refract Surg 2007; 33: 1826-1827.

9. Pärssinen O. The use of tamsulosin and iris hipotony during cataract surgery (letter). Acta Ophthalmol Scand 2005; 83: 625-626.

10. Pärssinen O, Leppänen E, Keski-Rahkonen P, Mauriala T, Dugué B, Lehtonen M. Influence of tamsulosin on the iris and its implacations for cataract surgery. Invest Ophthalmol Vis Sci 2006; 47: 3766-3771.

11. Cheung CM, Awan MA, Sandramouli S. Prevalence and clinical findings of tamsulosin-associated intraoperative floppy-iris syndrome. J Cataract Refract Surg 2006; 32: 1336-1339.

12. Schwinn DA, Afshari NA. $\alpha 1$-adrenergic receptor antagonists and the iris: new mechanistic insights into floppy iris syndrome. Surv Ophthalmol 2006; 51: 501-512.

13. Bendel RE, Philips MB. Preoperative use of atropine to prevent intraoperative floppy-iris syndrome in patients taking tamsulosin. J Cataract Refract Surg 2006; 32: 1603-1605.

14. Shugar JK. Prophylaxis for IFIS. J Cataratact Refract Surg 2007; 33: 942-943.

15. Gurbaxani A, Packard R. Intracameral phenylephrine to prevent floppy iris syndrome during cataract surgery in patients on tamsulosin. Eye 2005; 21: 331-332.

16. Gallenga PE, Lobefalo L. Postoperative finding in the intraoperative floppy-iris syndrome. J Cataract Refract Surg 2007; 33: 1811-1812.

17. Wilson ME, Trivedi RH, Mistr S. Pediatric intraoperative floppy-iris syndrome. J Cataract Refract Surg 2007; 33: 1325-1327.

18. Cionni RJ, Barros MG, Kaufman AH, Osher RH. Cataract surgery without preoperative eye drops. J Cataract Refract Surg 2003; 29: 2281-2283.

19. Lundberg B, Behndig A. Separate and additive mydriatic effects of lidocaine hydrochloride, phenylephrine, and cyclopentolate after intracameral injection. J Cataract Refract Surg 2008; 34: 280-283. 